\title{
Gastrocnemius tubercle: attachment point of the gastrocnemius muscle tendon and posterior oblique ligament
}

\begin{abstract}
Introduction: The gastrocnemius tubercle is a third bony prominence found in the medial inferior part of the femur, gently distal and posterior to the adductor tubercle. It was named after its close location to the depression corresponding to the insertion of the medial gastrocnemius tendon. The gastrocnemius tubercle is likely to provide an extra surface for the insertion of the medial head of the gastrocnemius muscle. The aim of this study was to verify the incidence of the gastrocnemius tubercle in bones of dry femurs in northeastern Brazil
\end{abstract}

Materials and methods: We evaluated 110 intact medial femoral condyles of human adults, found at the Human Anatomy Laboratories of the Federal University of Sergipe and the Tiradentes University, and 54 were right and 56 left, of unknown gender and age.

Results: Of the 110 medial femoral condyles evaluated, the gastrocnemius tubercle was found in $82.72 \%(91)$ of the cases. In the right dimidium this incidence was $41.8 \%(46)$ and in the left $40.9 \%(45)$

Conclusion: The gastrocnemius tubercle has a high incidence in the medial femoral condyles of human adults, and its recognition is of great importance to clinicians and orthopaedic surgeons in the diagnosis and treatment of injuries on the knee medial face.
Volume 6 Issue 6 - 2019

\author{
José Aderval Aragão, ${ }^{1,4}$ Julia Dória Fontes, ${ }^{2}$ \\ lapunira Catarina Sant' Anna Aragão, ${ }^{3}$ Felipe \\ Matheus Sant' Anna Aragão, ${ }^{3}$ Francisco Prado \\ Reis ${ }^{4}$ \\ 'Department of Morphology, Federal University of Sergipe \\ (UFS), Brazil \\ ${ }^{2}$ Department of Medicine, Federal University of Sergipe (UFS), \\ Brazil \\ ${ }^{3}$ Medical School, University Center of Volta Redonda (UNIFOA), \\ Brazil \\ ${ }^{4}$ Medical School of Tiradentes University (UNIT), Brazil
}

Correspondence: José Aderval Aragão, Federal University of Sergipe, Marechal Rondon Avenue, São Cristóvão, Sergipe, Brazil, Tel +55-79-991916767, Email adervlufs@gmail.com

Received: November 10, 2019 | Published: November 22 2019

Keywords: gastrocnemius tubercle, femur, incidence, referential

Abbreviations: MFC, medial femoral condyle; LFC, lateral femoral condyle; Red Circle, the gastrocnemius tubercle

\section{Introduction}

The gastrocnemius tubercle is a third prominence found in the medial inferior part of the femur, gently distal and posterior to the adductor tubercle. It was named after its close location to the depression corresponding to the insertion of the medial gastrocnemius tendon. ${ }^{1}$ The gastrocnemius tubercle, the adductor tubercle and the femoral medial epicondyle form a triangular space that houses the origin of the medial patellofemoral ligament ${ }^{2}$ and it is likely that the gastrocnemius tubercle provides an extra surface for the insertion of the medial head of the gastrocnemius muscle. ${ }^{3}$ Thorough anatomical knowledge of the medial part of the knee is indispensable for the diagnosis and treatment of injuries, ${ }^{4}$ as well as avoiding nonanatomical repairs to the medial part of the knee. ${ }^{1}$ The aim of this study was to verify the incidence of the gastrocnemius tubercle in bones of dry femurs in north-eastern Brazil.

\section{Materials and methods}

We evaluated 110 intact medial femoral condyles of human adults, found at the Human Anatomy Laboratories of the Federal University of Sergipe and the Tiradentes University, and 54 were right and 56 left, of unknown gender and age.

\section{Results}

Of the 110 medial femoral condyles, the gastrocnemius tubercle was found in $82.7 \%$ (91) of the cases (Figure 1). In the right dimidium this incidence was $41.8 \%$ (46) and in the left $40.9 \%$ (45) (Table 1).

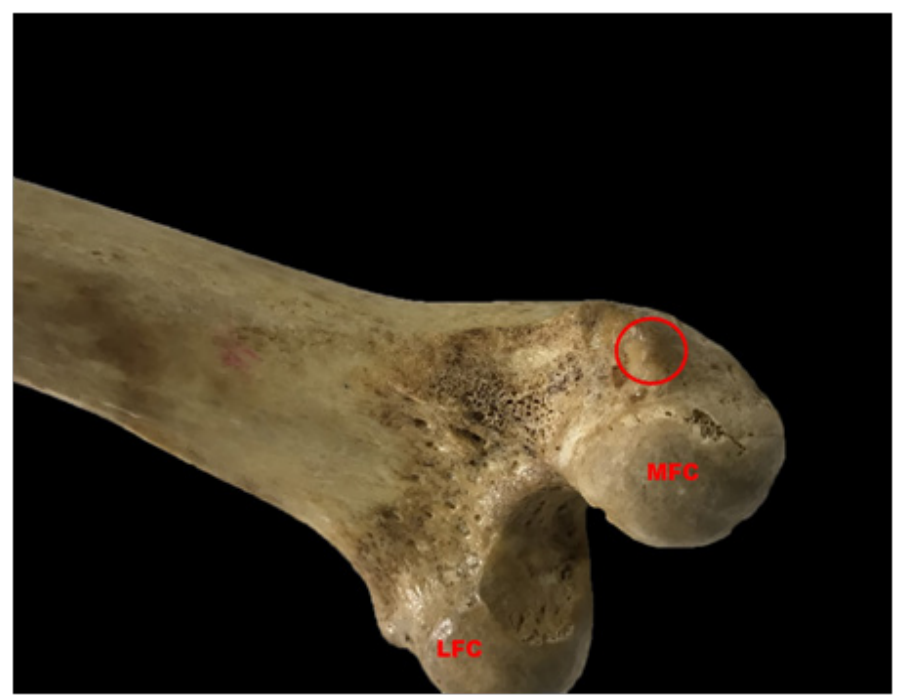

Figure I The gastrocnemius tubercle (red circle).

MFC, medial femoral condyle; LFC, lateral femoral condyle. 
Table I Incidence of the gastrocnemius tubercle according to the dimidium

\begin{tabular}{lllllll}
\hline Tubercle & \multicolumn{2}{l}{ Dimidium } & & & \\
& \cline { 2 - 7 } & \multicolumn{2}{l}{ Right } & & Left & \multicolumn{2}{l}{ Total } & \\
\cline { 2 - 7 } & Number & $\%$ & Number & $\%$ & Number & $\%$ \\
\hline Present & 46 & 41,8 & 45 & 40,9 & 91 & 82,7 \\
Absent & 8 & 7,3 & 11 & 10,0 & 19 & 7,3 \\
Total & $\mathbf{5 4}$ & $\mathbf{4 9 , 1}$ & $\mathbf{5 6}$ & $\mathbf{5 0 , 9}$ & $\mathbf{1 1 0}$ & $\mathbf{1 0 0 , 0}$ \\
\hline
\end{tabular}

\section{Discussion}

Further studies on the gastrocnemius tubercle bring values between $52.27 \%$ and $100 \%$ of occurrence, ${ }^{1,3}$ and in $80.7 \%$ of those which presented this prominence, it was smaller than the adductor tubercle. ${ }^{3}$ The authors of the present study identified an occurrence of $82.7 \%$, which corroborates the high percentages of occurrence found by LaPrade et al. ${ }^{1}$ \& Gosavi et al. ${ }^{3}$ detailed anatomical knowledge of the medial part of the knee is indispensable for the diagnosis and treatment of injuries, such as the sensitivity perceived through palpation of the adductor tubercle as an indicator for injury at the origin of the medial collateral ligament. ${ }^{4}$ While the adductor tubercle apex is readily identified on palpation and confirmed as a reference point for the medial collateral and the posterior oblique ligaments, the gastrocnemius tubercle apex is not so easily identified because of its variable and superficial shape. ${ }^{5}$ Despite this difference, recognition of the gastrocnemius tubercle is important so that clinicians do not confuse it with the adductor tubercle during palpation, resulting in non-anatomical repairs to the medial part of the knee, ${ }^{1}$ and to identify regions marked by chronic injuries or previous surgery. ${ }^{6}$

\section{Conclusion}

The gastrocnemius tubercle has a high incidence in the medial femoral condyles of human adults, being qualified as anatomical reference as well as the adductor tubercle and femoral medial epicondyle. Its recognition is of great importance to assist clinicians and orthopaedic surgeons in the diagnosis and surgical repair of the medial knee face.

\section{Acknowledgments}

We thank Eufrásio dos Santos Cardoso and Max Wesley Santos Hora, anatomy laboratory technicians in the Tiradentes University, for their support in preparing the skeleton, since without their collaboration, this work could not have been accomplished.

\section{Conflicts of interest}

Author declares there are no conflicts of interest.

\section{References}

1. LaPrade RF, Engebretsen AH, Ly TV, et al. The anatomy of the medial part of the knee. J Bone Joint Surg Am. 2007;89(9):2000-2010.

2. Aframian A, Smith TO, Tennent TD, et al. Origin and insertion of the medial patellofemoral ligament: a systematic review of anatomy. Knee Surg Sports Traumatol Arthrosc. 2017;25(12):3755-3772.

3. Gosavi S, Garud R, Jadhav S. Gastrocnemius tubercle in Indian population: a new anatomical entity? Rev Arg de Anat Clin. 2015;7(2):107-111.

4. Bonasia DE, Bruzzone M, Dettoni F, et al. Treatment of medial and posteromedial knee instability: indications, techniques, and review of the results. Iowa Orthop J. 2012;32:173-183.

5. Saigo T, Tajima G, Kikuchi S, et al. Morphology of the insertions of the superficial medial collateral ligament and posterior oblique ligament using 3-dimensional computed tomography: a cadaveric study. Arthroscopy. 2017;33(2):400-407.

6. Laprade RF, Wijdicks CA. The management of injuries to the medial side of the knee. J Orthop Sports Phys Ther. 2012;42(3):221-233. 